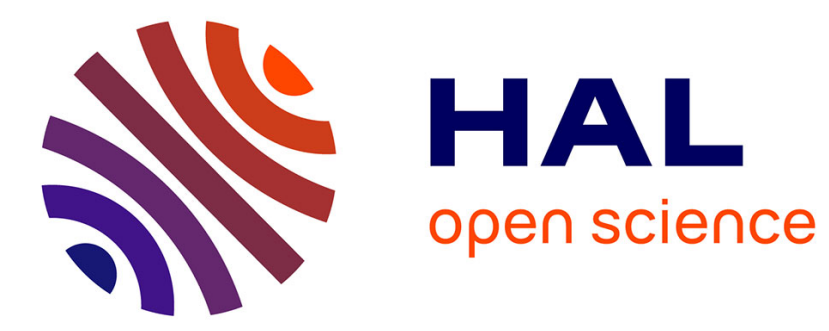

\title{
Sur la réflexion métallique des rayons calorifiques obscurs polarisés
}

\author{
L. Mouton
}

\section{To cite this version:}

L. Mouton. Sur la réflexion métallique des rayons calorifiques obscurs polarisés. J. Phys. Theor. Appl., 1878, 7 (1), pp.157-168. 10.1051/jphystap:018780070015701 . jpa-00237390

\section{HAL Id: jpa-00237390 https://hal.science/jpa-00237390}

Submitted on 1 Jan 1878

HAL is a multi-disciplinary open access archive for the deposit and dissemination of scientific research documents, whether they are published or not. The documents may come from teaching and research institutions in France or abroad, or from public or private research centers.
L'archive ouverte pluridisciplinaire HAL, est destinée au dépôt et à la diffusion de documents scientifiques de niveau recherche, publiés ou non, émanant des établissements d'enseignement et de recherche français ou étrangers, des laboratoires publics ou privés. 


\section{SUR LA RÉFLEXION MÉTALLIQUE DES RAYONS GALORIFIQUES OBSGURS POLARISÉS;}

PAR M. L. NOUTON.

Avant de résumer ce travail $\left({ }^{2}\right)$, il n'est pas inutile, je crois, d'énoncer les lois relatives à la réflexion de la lumière, qui résultent surtout des études expérimentales de M. Jamin:

I. Rappel des lois relatives à la réflexion de la lumière.$I^{\circ} \mathrm{Si}$ l'on fait réfléchir un nombre quelconque de fois sur un miroir quelconque un rayon polarisé dans les azimuts déterminés par le plan d'incidence et le plan perpendiculaire (azimuts principaux), il reste toujours polarisé dans le même plan après la réflexion.

Ce fait a été établi expérimentalement par Brewster pour le verre et les métaux $\left({ }^{3}\right)$; bien qu'il découle naturellement de la loi de symétrie appliquée à l'idée qu'avec Fresnel nous nous faisons d'un rayon polarisé, il n'en doit pas moins être placé en tête de toute étude purement expérimentale des phénomènes de la réflexion.

( $\left.{ }^{1}\right)$ Annales de Chimie et de Physique, $4^{\mathrm{e}}$ sé:ie, t. XVI, p. 86.

( $\left.{ }^{2}\right)$ Voir Annales de Chimie et de Physique, 5e séiie, 1. XIII, p. 229 et siiv.

( $\left.{ }^{3}\right)$ Annales de Chimie et de Physique, $3^{\mathbf{e}}$ série, t. XIX, p. 296.

J. de Phys., t. VII. (Mai ı 3,8.) 
Combiné avec le principe de la coexistence des petits mouvements, il constitue en effet la base de tous les raisonnements et de toutes les méthodes usités dans ce genre de recherches. Eux établis, pour étudier l'action de la réflexion sur un rayon primitivement polarisé dans un azimut quelconque, on le décompose en deux, polarisés dans les azimuts principaux; la direction de leurs vibrations n'ayant pas été changée par la réflexion, ils n'ont pu éprouver que des changements de phase et des variations d'amplitude.

Les variations d'amplitude peuvent s'exprimer par la multiplication des amplitudes incidentes par deux nombres I (vibration parallèle au plan d'incidence) et $\mathbf{J}$ (vibration perpendiculaire à ce plan), ces deux nombres étant supposés toujours positifs et évidemment au plus égaux à l'unité.

Un changement quelconque produit dans la phase d'un mouvement vibratoire peut toujours être considéré comme résultant d'un certain chemin parcouru dans l'air; le phénomène de la réflexion peut ainsi s'assimiler à la traversée d'une lame cristalline, traversée qui, convertie en air, donne un chemin $d$ pour la vibration parallèle au plan d'incidence, et un chemin $d^{\prime}$ pour l'autre.

Le nombre $\frac{d-d^{\prime}}{\lambda}$ est la différence de phase produite par la réflexion pour une lumière de longueur d'onde $\lambda$. Par raison de symétrie, il est évidemment nul pour l'incidence normale $\left({ }^{1}\right)$.

Les recherches ont surtout porté sur la détermination pour les diverses incidences du rapporl $\frac{\mathbf{I}}{\mathbf{J}}$ et du nombre $\frac{d-d^{\prime}}{\lambda}$.

( ${ }^{1}$ Il se produit ici, à l'observation, un fait particulier qu'il faut signaler. Par suite du retournement de l'observateur, qui se place naturellement de façon à recevoir dans l'œil le rayon réfléchi comme il y recevait le rayon incident, la vibration située dans le plan d'incidence paraît aroir changé de signe, ou si l'on aime mieux avoir subi un retard d'une demi-onde. Ainsi, à l'incidence normale, un rayon primitivement polarisé dans un azimut quelconque paraît l'ètre après la réflexion dans l'azimut symétrique par rapport aux plans principaux; c'est que l'observateur s'est retourné, et qu'il n'en continue pas moins à définir par sa droite ou sa gauche la direction positive de l'axe de coordonnées situé dans le plan d'incidence. Je crois qu'il est naturel de ne pas faire entrer cette demi-longueur d'onde dans l'expression physique du phénomène; la différence de phase est alors bien nulle à l'incidence normale, et les résultats d'observation ainsi modifiés se trouvent immédiatement comparables â ceux que fournissent les théories mécaniques, où naturellement on conserve toujours le mème système d'axes de coordonnées. 
Voici les faits généraux qui se sont trouvés ajoutés à la loi de Brewster énoncée plus haut:

$2^{\circ}$ La différence de phase partant de o à l'incidence normale atteint pour tous les corps la valeur $\frac{1}{2}$ à l'incidence rasante ou de 9 o degrés; cette valeur est positive pour les métaux et les substances transparentes d'indice supérieur à $\mathrm{I}, 46$ environ;

$3^{\circ}$ La forme de la courbe représentative de ces valeurs (des longueurs proportionnelles aux angles d'incidence étant prises pour abscisses) diffère avec les substances réfléchissantes; ainsi, pour les métaux, elle paraît s'élever d'une façon continue depuis l'incidence o jusqu'à $90^{\circ}$; pour le verre, elle reste sensiblement confondue avec l'axe des abscisses jusqu'à l'incidence brewstérienne ou de polarisation; puis, s'élevant brusquement, elle atteint une valeur sensiblement égale à sa limite supérieure ${ }_{2}^{1}$;

$4^{\circ}$ Pour une même substance réfléchissante, la forme de la courbe se modifie d'une façon continue, quand la longueur d'onde de la lumière réfléchie se modifie elle-même d'une façon continue;

$5^{\circ}$ Les valeurs du rapport $\frac{\mathrm{I}}{\mathrm{J}}$, partant évidemment de l'unité pour l'incidence normale, paraissent y revenir pour l'incidence rasante;

$6^{\circ} \mathrm{La}$ forme de la courbe qui représente ces valeurs est également propre à chaque substance et à chaque longueur d'onde;

$7^{\circ}$ Quelle que soit la longueur d'onde, la valeur $\underset{\mathbf{J}}{\mathbf{I}}$ passe par un minimum à l'incidence pour laquelle la différence de phase est 0,25 , ou, comme on dit souvent, la différence des retards $\left(d-d^{\prime}\right)$, un quart d'onde.

Pour bien comprendre les avantages de cette manic̀re d'envisager les phénomènes, rapportons-y d'abord les résultats classiques de Fresnel relatifs au verre. Fresnel obtient pour les deux coefficients des amplitudes $-\frac{\sin (i-r)}{\sin (i+r)}$ et $-\frac{\operatorname{tang}(i-r)}{\tan g(i+r)}$, et aucune différence de phase, ce qui n'a rien de surprenant, puisqu'il n'en suppose pas tout d'abord.

Dans la façon de voir proposée plus haut, nous devrons prendre pour I et $J$ les valeurs positives "des nombres $\frac{\sin (i-r)}{\sin (i+r)}$ et $\frac{\operatorname{tang}(i-r)}{\operatorname{tang}(i+\boldsymbol{r})} ;$ par suite, la courbe des rapports $\frac{\mathbf{I}}{\mathbf{J}}$ est donnée par 
$\mathbf{I}=\frac{\cos (i+r)}{\cos (i-r)}$, le deuxième membre étant toujours pris avec sa valeur absolue positive. On voit que le rapport $\frac{\mathbf{I}}{\mathbf{J}}$ part de $\mathbf{r}$ à l'incidence normale, y revient à l'incidence rasante, et passe par un minimum qui est zéro pour $i+r=90^{\circ}$ : c'est l'angle de polarisation ou incidence brewstérienne. Laction de la longueur d'onde se fait sentir par la présence simultanée des angles $i$ et $r$.

Quant à la courbe des différences de phase, elle se confond avec l'axe des incidences jusqu'à $i \leftarrow r=90^{\circ}$; là, elle s'élève en une droite $\mathrm{AB}$ normale à l'axe des abscisses jusqu'à une valeur $\frac{1}{2}$, où elle s'achève par une parallèle à cet axe. On voit sans peine que cette différence d'une demi-onde vient remplacer le changement de signe du terme $\frac{\operatorname{tang}(i-r)}{\operatorname{tang}(i+r)}$.

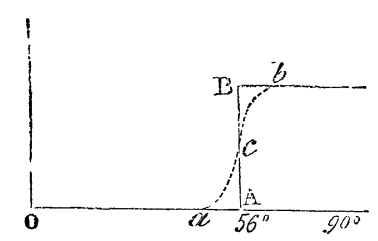

Mais il va nous être facile de comprendre ce qu'ont ajouté à ces résultats les expériences précises de M. Jamin.

Les coefficients de Fresnel sont trouvés exacts, mais les angles $\mathbf{A}$ et $\mathrm{B}$ de la courbe des différences de phase doivent être adoucis, et la différence brusque d'une demi-onde doit être remplacée par une marche rapide, mais continue, grossièrement représentée par la ligne ponctuée $a b$; el c'est au point $c$, passage par 0,25 , que correspond l'incidence brewstérienne, minimum zéro du rapport $\frac{\mathbf{I}}{\mathbf{J}}$.

La réflexion sur le verre et les corps transparents ne diffère ainsi que par la forme des courbes qui la caractérisent de la réflexion sur les métaux.

Je me suis proposé de rechercher comment se modifient les lois précédentes, quand, sortant du spectre lumineux, on fait réfléchir 
sur des miroirs métalliques des radiations obscures purement calorifiques, occupant, dans le spectre général, des positions bien déterminées de plus en plus distantes du rouge.

Je résumerai successivement la disposition expérimentale, le mode d'opération et de calcul, et les résultats que j'ai déjà obtenus.

II. Disposition expérimentale. - La source de chaleur est la lampe de MM. Bourbouze et Viesnegg, dans laquelle, comme on le sait, un capuchon de toile de platine est maintenu au rouge blanc par la combustion du gaz d'éclairage et d'un courant d'air amené par une trompe à eau à une pression constante d'environ 20 centimètres de mercure. Dans ces conditions, cette source est d'une remarquable constance, et elle présente de plus l'avantage d'un grand développement du spectre calorifique obscur.

La lampe est placée dans une première pièce à $3 o$ centimètres environ de la cloison qui sépare cette pièce de la voisine: cette cloison est percée d'un trou où est encastrée, à hauteur de la lampe, une lentille de verre ordinaire de r 5 centimètres de foyer. Dans la seconde pièce, sur un solide banc d'optique articulé, se trouvent : $\mathrm{I}^{\circ}$ un énorme prisme biréfringent avec alidade et cercle divisé, à image extraordinaire redressée et achromatisée : c'est le polariseur $; 2^{\circ}$ une plaque de verre à inclinaison variable et mesurée autour d'un axe horizontal, destinée à compenser les effets de polarisation produits par le piprisme disperseur qu'on va voir $\left({ }^{1}\right)$; $3^{\circ}$ un écran percé d'une fente sur laquelle se forme l'image extraordinaire de la lampe; l'image ordinaire, en tournant, est, dans toutes les orientations du polariseur, interceptée par l'écran; plus loin, se trouve une lentille; puis, au centre d'une articulation du banc, le miroir sur un cercle gradué mesurant l'incidence. Le faisceau réfléchi est reçu sur un prisme de flint blanc, puis traverse un nouveau prisme biréfringent, analyseur semblable au polariseur, et se résout finalement en deux spectres dont l'extraordinaire demeure fixe et pur pendant la rotation de l'analyseur, l'ordinaire tournant autour sans jamais empiéter sur lui. Le spectre extraordinaire tombe sur une pile thermo-électrique linéaire, dont l'ou-

(') J'ai emprunté l'idée de cette lame compensatrice au travail de MM. Fizeau et Foucault (Annales de Chimie et de Physique, $3^{\mathrm{e}}$ série, t. XXX, p. 14́47). 
verture est, comme cclle de la fente, de I millimètre environ, communiquant avec un excellent galvanomètre de M.Ruhmkorff ( $\left.{ }^{1}\right)$. Cette pile était munie d'une vis permettant de la déplacer paral-. lèlement aux bandes du spectre.

III. Mode d'opération et de calcul. — J'ai opéré sur trois longueurs d'onde que je désigne par $\lambda_{1}, \lambda_{2}, \lambda_{3}$, réparties dans la partie obscure du spectre, et sensiblement symétriques par rapport au rouge extrême, $\lambda_{1}$ du jaune, $\lambda_{2}$ du vert bleu et $\lambda_{3}$ du bleu indigo. Ayant été témoin d'une partie des expériences par lesquelles M. Desains a étudié la rotation imprimée par une plaque de quartz perpendiculaire aux plans de polarisation des rayons calorifiques obscurs $\left({ }^{2}\right)$, j'ai pu, par le même procédé, fixer cette rotation pour les trois longueurs d'onde que j'ai employées. Avec une plaque de quartz droit, qui donnait à la lumière du sodium une rotation exacte de $\mathbf{~}$ oo degrés, les rotations correspondant aux radiations $\lambda_{1}, \lambda_{2}, \lambda_{3}$ ont été respectivement 32 , r6 et ro degrés. En prenant la loi de Biot, au moins comme première approximation, on obtiendrait

$$
\lambda_{1}=\mathrm{o}^{\mathrm{mm}}, \text { oо10 }, \quad \lambda_{2}=\mathrm{o}, 00 \mathrm{1} 4, \lambda_{3}=0,0018
$$

ces chiffres, dont le dernier est supérieur au triple de la longueur d'onde des raies $\mathrm{D}$, donnent une idée du secours qu'on est en droit d'attendre de l'étude des phénomènes calorifiques au point de vue de l'établissement ou de la vérification des théories optiques.

Je n'avais pas à démontrer le principe de Brewster relativement aux rayons calorifiques obscurs. Dès 1849 , MM. de la Provostaye et P. Desains étudiaient $\left({ }^{3}\right)$ ce que devenaient, après leur réflexion, des rayons calorifiques primitivement polarisés dans l'un ou l'autre des azimuts principaux, constataient que la réllexion ne modifie pas leur plan de polarisation et établissaient même les lois de la variation de leur intensité avec lincidence. Aussi, dans toutes les séries d'expériences, la vérification de cette première loi était mon

( ${ }^{1}$ ) On arrive facilement à apprécier le $\frac{1}{10}$ de deģré, ce quii suffit; le galvanomètre n'étant jamais dans un repos absolu, quelques soins que l'on prenne d'éviter les mouvements de l'air autour de la pile, les méthodes d'amplification des angles ne présentent ici, le plus souvent, que des avantages illusoires.

( ') Comptes rendus de l'Académie des Sciences, 1'́l mai 1 877, p. 105̄6.

$\left({ }^{3}\right)$ Annales de Chimie et de Physique, $3^{\text {e }}$ série, t. XXVII, p. rog et suiv. 
critérium du bon réglage des divers appareils et de l'état du galvanomètre, les déviations de celui-ci devant, dans les différents azimuts, indiquer des intensités conformes à la loi de Maıus.

Quand le rayon incident est polarisé dans un azimut autre que zéro ou $9^{o}$ degrés, la réflexion le rend en général elliptique. C'est donc aux propriétés d'un tel rayon qu'on doit demander les procédés expérimentaux destinés à fournir le rapport $\frac{\mathbf{I}}{\mathbf{J}}$ et la différence de phase $\frac{d-d^{\prime}}{\lambda}$ définis plus haut.

Je ne ferai qu’énoncer ici celles de ces propriétés sur lesquelles je me suis appuyé, renvoyant pour leur démonstration aux Études sur la réflexion métallique de la lumière de M. Jamin ( $\left.{ }^{1}\right)$.

Le plan d'incidence est horizontal; l'observateur recevant le rayon réfléchi en pleine poitrine, je prends l'axe des $x$ dans le plan d'incidence, et la partie positive de cet axe toujours dirigée vers la droite de l'observateur, supposé inconscient du demi-tou qu'il a opéré; l'axe des $y$ est perpendiculaire au précédent et se dirige de bas en haut; les angles seront comptés, comme d'habitude, de $o x$ vers $o y$.

$\mathrm{I}^{\mathrm{o}}$ Mesure de $\frac{\mathbf{l}}{\mathbf{J}}$. Si la vibration incidente fait un angle $a$ avec l'axe des $x$, ses deux composantes ont pour amplitudes $\cos a$ et $\sin a$, et après la réflexion $\mathrm{I} \cos a$ et $\mathbf{J} \sin a$. L'analyseur placé successivement dans les azimuts o et 90 degrés, on aura deux lectures galvanométriques proportionnelles à $\mathrm{J}^{2} \cos ^{2} a$ et $\mathrm{J}^{2} \sin ^{2} a$. Bien que, par les vérifications de la loi de Malus dont j'ai parlé tout à l'heure, je fusse assuré de la proportionnalité des déviations galvanométriques aux intensités calorifiques dans les limites où j'opérais, j'ai préféré toujours disposer de l'angle $a$ dans cette première mesure, de façon à rendre à peu près égales les deux lectures.

Si $\alpha$ et $\beta$ sont ces deux déviations, on a

$$
\mathbf{I}=\operatorname{tang} a \sqrt{\frac{\alpha}{\beta}}
$$

(') Annales de Chimie et de Physique, $3^{\circ}$ série, t. XIX, p. $32 \mathrm{I}$ et suiv. 
$2^{0}$ Mesure de $\frac{d-d^{\prime}}{\lambda}$. Après avoir traversé un prisme- de spath dont on n'utilise que l'image extraordinaire, un rayon elliptique présente, quand la section principale du prisme coïncide avec le grand axe de l'ellipse, un maximum d'intensité; avec le petit axe, un minimum; et, si l'on mesure ces intensités dans des couples d'azimuts $\alpha$ et $\alpha+90^{\circ}$, en allant du grand axe au petit, la première l'emportera sur la seconde, tant que l'azimut $\alpha$ sera compris entre le grand axe et 45 degrés de cet axe, pour lui devenir inférieure dès que $\alpha$ aura dépassé cette bissectrice des axes.

On peut donc, par ce moyen, fixer l'azimut des bissectrices des axes de l'ellipse, et on le peut d'autant mieux que c'est autour de ces bissectrices qu'une même variation $\Delta x$ produit dans les deux lectures rectangulaires une plus grande différence. J'ai pu, dans les bonnes séries, le fixer à $\frac{1}{2}$ degré près.

A ces avantages, déjà utilisés par de Senarmont pour la lumière, s'en joignent de spéciaux aux études calorifiques: d'abord il n'est demandé à la source de chaleur que d'ètre constante pendant la durée de chaque couple d'observations, et j'ai pu rendre cette durée très-courte par un mouvement spécial avec buttage, permettant de faire tourner rapidement l'analyseur de go degrés sans l'approcher de la pile; en second lieu, les mesures se terminent par deux valeurs égales des dévialions galvanométriques : c'est un avantage qu'apprécieront ceux qui ont eu occasion de se servir de galvanomètres très-sensibles.

L'azimut desbissectrices desaxes del'ellipse étant ainsi déterminé, on en tire l'azimut $\omega$ des axes eux-mêmes en ajoutant ou retranchant 45 degrés. On peut, d'ailleurs, faire autant de mesures qu'on le veut, en faisant varier l'azimut de polarisation de la vibration incidente.

$$
\text { Cela fait, si l'on pose } \frac{\mathrm{I} \cos a}{\mathrm{~J} \sin a}=\cot \alpha \text {, }
$$
et

on a

$$
2 \pi\left(\frac{1}{2}-\frac{d-d^{\prime}}{\lambda}\right)=\delta
$$

$$
\cos \delta=\frac{\operatorname{tang} 2 \omega}{\operatorname{tang} 2 \alpha}
$$

qui détermine $\delta$ et par suite $\left.\frac{d-d^{\prime}}{\lambda}{ }^{1}\right)$.

(1) Le lecteur familiarisé avec les études de de Sénarmont et de M. Jamin etablira 
IV. Résultats obtenus. - J'ai opéré jusqu'à présent sur trois miroirs d'acier, de métal des miroirs et de verre platiné. Les tableaux suivants résument les résultats que j’ai obtenus :

\section{Acier.}

$\begin{array}{ll}\text { Différences de } & \\ \text { Incidences. } & \text { phases } \frac{c-l^{\prime}}{\zeta} .\end{array}$

Longueur d'onde $\lambda_{1}$.

$\begin{array}{llcc}45^{\circ} & \ldots \ldots \ldots \ldots \ldots & \text { Insensible. } & 0,86 \\ 50 & \ldots \ldots \ldots \ldots & 0,02 & 0,84 \\ 55 & \ldots \ldots \ldots \ldots & 0,03 & 0,82 \\ 60 & \ldots \ldots \ldots \ldots & 0,05 & 0,80 \\ 65 & \ldots \ldots \ldots \ldots & 0,09 & 0,76 \\ 70 & \ldots \ldots \ldots \ldots & 0,19 & 0,70 \\ 75 & \ldots \ldots \ldots \ldots & 0,26 & 0,62 \\ 79 & \ldots \ldots \ldots \ldots & 0,31 & 0,53 \\ 80 & \ldots \ldots \ldots \ldots \ldots & 0,55 \\ 82 & \ldots \ldots \ldots \ldots & 0,58\end{array}$

Longueur d'onde $\lambda_{2}$.

50

$\begin{array}{rr}0 & 0,88 \\ 0,01 & 0,85 \\ 0,03 & 0,78 \\ 0,06 & 0,75 \\ 0,10 & 0,64 \\ 0,15 & 0,62 \\ 0,20 & 0,55 \\ 0,22 & 0,5 \text { r } \\ 0,25 & 0,51 \\ 0,29 & 0,55\end{array}$

sans peine cette formule. On trouvera dans le Mémoire (Annales de Chimie et de Physique, $5^{\mathrm{e}}$ sćrie, t. XIII, p. 239) dont je donne ici un extrait la discussion relative au choix à faire parmi l'infinité d'ares ayant même cosinus et le calcul complet d'une expérience. 
Acier.

Incidences.

Différences de

phases $\frac{d-d^{\prime}}{i}$.

Rapport $\frac{1}{\mathbf{j}}$

Longueur d'onde $\lambda_{3}$.

\begin{tabular}{|c|c|c|c|}
\hline 50 & $\ldots \ldots \ldots \ldots$ & o & $0,9^{\circ}$ \\
\hline $6 o$ & $\ldots \ldots \ldots \ldots$ & 0,0 I & $o, 80$ \\
\hline $7^{5}$ & $\ldots \ldots \ldots \ldots$ & $0,1_{2}$ & o,6o \\
\hline 80 & $\ldots \ldots \ldots \ldots$ & 0,18 & 0,55 \\
\hline 82 & $\ldots \ldots \ldots \ldots$ & 0,22 & $0,5 \mathbf{r}$ \\
\hline 83,5 & $\ldots \ldots \ldots \ldots$ & 0,25 & o, 49 \\
\hline
\end{tabular}

Métal des miroirs.

Longueur d'onde $\lambda_{1}$.

\begin{tabular}{|c|c|c|c|}
\hline $60^{\circ}$ & $\ldots \ldots \ldots \ldots$ & $c, 08$ & o,88 \\
\hline 65 & $\ldots \ldots \ldots$ & $0,1 \mathrm{I}$ & 0,80 \\
\hline 70 & $\ldots \ldots \ldots$ & $0, \mathbf{1} \tilde{5}$ & 0,70 \\
\hline $7^{5}$ & $\ldots \ldots \ldots$ & 0,20 & $0,6 r$ \\
\hline 80 & $\ldots \ldots \ldots$ & $o, 30$ & 0,65 \\
\hline \multicolumn{4}{|c|}{ Longueur d'onde $\lambda_{2}$. } \\
\hline 65 & $\ldots \ldots \ldots \ldots$ & o, o6 & o, 84 \\
\hline 70 & $\ldots \ldots \ldots \ldots$ & 0,10 & 0,78 \\
\hline 75 & $\ldots \ldots \ldots \ldots$ & 0,16 & 0,70 \\
\hline 8o & $\ldots \ldots \ldots \ldots$ & 0,26 & $o, \dot{t i}_{2}$ \\
\hline
\end{tabular}

Verre platiné.

Longueur d'onde $\lambda_{2}$.

$\begin{array}{llll}65 & \ldots \ldots \ldots \ldots & 0,07 & 0,72 \\ 70 & \ldots \ldots \ldots \ldots & 0,12 & 0,54 \\ 75 & \ldots \ldots \ldots \ldots & 0,19 & 0,48 \\ 80 & \ldots \ldots \ldots \ldots & 0,27 & 0,46\end{array}$

Si l'on examine chacun des tableaux précédents, on y voit d'abord se reproduire les résultats généraux que j’ai rappelés plus haut, relatifs à la lumière, à savoir :

$\mathbf{I}^{\circ}$ Les différences de phases partant de o s'élèvent progressivement à mesure que s'accroît l'angle d'incidence; 
$2^{\circ}$ La valeur de $\frac{\mathbf{I}}{\mathbf{J}}$ partant de $\mathrm{I}$ à l'incidence normale va en diminuant, passe par un minimum, puis tend de nouveau vers l'unité; $3^{\circ}$ La valeur minimum du rapport $\frac{\mathbf{I}}{\mathbf{J}}$ correspond toujours à l'incidence pour laquelle $\frac{d-d^{\prime}}{\lambda}=0,25$.

Si maintenant, pour un même miroir, on comparc les séries des différences de phase correspondant aux diverses longueurs d'onde, il s'en dégage un enseignement qui a peut-être quelque importance.

Je vais mettre en regard, dans le tableau suivant, pour l'acier, les valeurs successives des différences de phases relatives à la longueur d'onde du thallium ( $\mathrm{o}^{\mathrm{mm}}$, ooo534, Mascart), du sodium $\left({ }^{1}\right)$ $\left(\mathbf{o}^{\mathrm{mm}}\right.$, ooo5888), et celles qui correspondent aux longueurs d'onde plus grandes encore $\lambda_{1}, \lambda_{2}, \lambda_{3}$, dont on a vu plus haut les valeurs approchées :

\begin{tabular}{|c|c|c|c|c|c|c|}
\hline $\begin{array}{c}\text { Incidences. } \\
0 \\
\end{array}$ & & Thallium. & $\begin{array}{l}\text { Sodium. } \\
\text { o, } 000588 \text {. }\end{array}$ & $\begin{array}{c}j_{1} \\
0,0010 .\end{array}$ & $\begin{array}{c}\partial_{2} \\
0,0014 .\end{array}$ & $\begin{array}{c}\lambda_{3} \\
0,0018 .\end{array}$ \\
\hline & $\cdots$ & 0,040 & oo, 3 o & o & o & $\mathbf{o}$ \\
\hline $4^{5}$ & $\cdots$ & o,o6̇o & 0,045 & o & o & o \\
\hline 50 & $\cdots$ & o, o8o & 0,065 & 0,02 & o & o \\
\hline 55 & $\cdots$ & 0,100 & o, o85 & o, o3 & $\mathbf{O}, \mathrm{OI}$ & o \\
\hline $6 o$ & $\ldots$ & $0,+15$ & 0, ro5 & 0,05 & o,o3 & $0, O_{I}$ \\
\hline 65 & $\cdots$ & $o, 155$ & 0,145 & 0,09 & o, o6 & ) \\
\hline 70 & $\cdots$ & 0,200 & 0,185 & $o, 15$ & 0,10 & » \\
\hline 75 & $\cdots$ & $o, 250$ & 0,240 & o, I 9 & 0,15 & $0, \mathrm{I} 2$ \\
\hline 76 & $\cdots$ & D & $o, 250$ & $"$ & 》 & 》 \\
\hline 79 & $\cdots$ & 》 & ") & 0,25 & ) & 》 \\
\hline 80 & $\cdots$ & 0,320 & o, 3 го & 0,26 & 0,20 & 0,18 \\
\hline 82 & $\cdots$ & " & " & $0,3 \mathbf{r}$ & 0,25 & 0,22 \\
\hline 83,5 & $\ldots$ & ) & ) & ) & ) & 0,25 \\
\hline
\end{tabular}

Ce tableau dessine nettement la marche du phénomène au moins

(1) Ces mesures ont été faites avec l'appareil et le compensateur de M. Jamin, construits par M. Duboscq. La source lumineuse était un brûleur Laurent avec sel marin fondu pour le sodium, et des grains d'alun de thallium pour le thallium. Une cuve remplie d'une dissolution de chlorure de cuivre arrêtait la raie jaunâtre du thallium, et surtout celle du sodium, qu'il est si difficile d'éviter. 
en ce qui concerne l'acier. Ainsi la différence de phase $\frac{d-d^{\prime}}{\lambda}$ est, à une incidence de 40 degrés, o,oło pour la lumière du thallium, $\mathrm{o}, \mathrm{o}$ o seulement pour le sodium, et insensible pour les radiations obscures. A 5o degrés, tandis qu'on a déjà o,o8o pour le vert, 0,065 pour le jaune, on commence à avoir $\mathbf{o}, 02$ pour $\lambda_{1}$ et rien encore pour les deux autres.

La différence de phase ne devient appréciable qu'à 55 degrés pour $\lambda_{2}$, à 60 degrés pour $\lambda_{3}$. Aussi, tout en se tenant inférieures, les différences de phasesrelatives aux plus grandes longueurs d'onde croissent-elles avec l'incidence d'une façon d'autant plus rapide que ces longueurs d'onde sont plus grandes.

Si l'on considère en particulier le point où la différence de phase est 0,25 , ou autrement dit où la différence de marche est $\frac{\lambda}{4}$, incidence de polarisation rétablie après deux réflexions, comme la désigne M. Jamin, on le trouve à $7^{5}$ degrés pour le vert, à 76 degrés pour le jaune, à 79 degrés pour $\lambda_{1}, 82$ degrés pour $\lambda_{2}$, $83 \frac{1}{2}$ degrés pour $\lambda_{3}$.

Des faits analogues se constatent dans les deux autres miroirs que j'ai étudiés.

Ainsi, pour ces corps, la courbe représentative des différences de phases reste d'autant plus longtemps confondue avec l'axe des incidences que la longueur d'onde est plus grande; par suite, l'intervalle est d'autant plus long pendant lequel la réflexion produira simplement une rotation du plan de polarisation incidente, tandis que d'autant plus court se trouve celui dans lequel la lumière ou la chaleur réfléchie sera polarisée elliptiquement. 